\title{
A batch processing protocol for construction of expression vector plasmids from a cDNA collection and Agrobacterium-mediated transformation of suspension-cultured cells of Arabidopsis
}

\author{
Yuki Naito ${ }^{\dagger}$, Yayoi Tsujimoto-Inui ${ }^{a, \dagger}$, Toshitsugu Nakano, Hideaki Shinshi, \\ Kaoru Suzuki* \\ Institute for Biological Resources and Functions, National Institute of Advanced Industrial Science and Technology \\ (AIST), Tsukuba Central 6, Tsukuba, Ibaraki 305-8566, Japan \\ *E-mail: kaoru-suzuki@aist.go.jp Tel: +81-29-861-6071 Fax: +81-29-861-6090
}

Received February 16, 2009; accepted June 8, 2009 (Edited by M. Sekine)

\begin{abstract}
In order to accelerate functional analysis of large transcription factor families, development of a simple and easy protocol to generate transgenic cells of Arabidopsis overexpressing the transgenes using collections of cDNAs for coding sequences (CDSs) of the family genes is promising. We conceived a batch processing protocol for construction of plant expression vector plasmids and Agrobacterium-mediated transformation of suspension-cultured cells using a Gateway system-compatible cDNA collection and demonstrated its feasibility using a cDNA collection of the DOF family. The present results suggested that the batch processing from the LR reaction to the transformation of Agrobacterium cells was properly performed. The specific overexpression of a single DOF transgene was observed in at least 6 of 8 lines, and a transgenic callus obviously overexpressing multiple DOF transgenes was not obtained. These results show the potential utility of the present protocol to gain a clue for detailed functional analyses of Arabidopsis genes.
\end{abstract}

Key words: Agrobacterium-mediated transformation, a batch processing protocol, Gateway system, suspension-cultured cells.

Understanding of function of transcription factors is an important step towards to develop the transcription factor-based technology for metabolic engineering and improvement of value and productivity of plants (Century et al. 2008; Grotewold 2008; Qu and Zhu 2006; Zhang 2003). Since it became possible to identify putative genes for transcription factors on a genomewide scale and it was revealed that most transcription factors could be grouped into large gene families with the completion of the Arabidopsis sequences (Arabidopsis Genome Initiative 2000; Riechmann et al. 2000), databases of transcription factors of Arabidopsis have been established on the basis of classification into gene families (Davuluri et al. 2003; Guo et al. 2008; Riano-Pachon et al. 2007), and the functional analysis of transcription factor families in Arabidopsis has been eagerly undertaken (e.g. Bi et al. 2005; Overvoorde et al. 2005; Lee et al. 2008; Tsujimoto-Inui et al. 2009). However, the most of transcription factor genes, which has been estimated to be $\sim 2000$ in Arabidopsis genome, has been remained to be characterized at the functional level (Qu and Zhu 2006).

The overexpression strategy appears particularly effective based on the unique characteristics and modes of action of transcription factors (Zhang 2003), since overexpression of transgenes may confer dominant gain-of-function phenotypes and sometimes confers unexpected beneficial traits. Such beneficial traits by overexpression are easily applied to genetic engineering in economically, agriculturally, and/or ecologically important plant species.

Suspension-cultured cells have been often used as simplified experimental systems in plant, since they are advantageous for collecting large amount of homogeneous cells and for reducing the complexity of plant tissues facilitating obtaining reproducible results. Several cell lines of suspension-culture of Arabidopsis, which is a favorable model plant for wide range of functional genomics studies, have been established. Among them, a unique cell line, namely, T87, which has photosynthetic ability under light irradiation (Axelos et al. 1992), have been widely used for biochemical and

Abbreviations: CaMV35S, cauliflower mosaic virus 35S; CDS, coding sequence; DOF, DNA-binding with one finger; EtBr, ethidium bromide; GUS, $\beta$-galacturonidase; PCR, polymerase chain reaction; RT-PCR, reverse transcriptase-mediated polymerase chain reaction.

${ }^{a}$ Present address: Biotechnology Research Center, The University of Tokyo, Tokyo, 113-8657, Japan

$\dagger$ These authors contributed equally to this work.

This article can be found at http://www.jspcmb.jp/ 
molecular biological analyses with respect to metabolic regulation, circadian rhythm, and stress response (e.g. Callard et al. 1996; Nakamichi et al. 2004; Nakamura et al. 2007; Stolc et al. 2005; Takahashi et al. 2001; Uno et al. 2000). Thus, this culture system is suitable for functional genomics of transcription factors.

Taken together, development of simple and easy protocols to generate transgenic cultured cells of Arabidopsis overexpressing transcription factors, which may be subsequently used for functional screenings, using collections of cDNAs for coding sequences (CDSs) is promising in order to accelerate functional analysis of large families. To this end, we conceived a batch processing protocol for construction of plant expression vector plasmids and Agrobacterium-mediated transformation of Arabidopsis suspension-cultured cells using a Gateway system-compatible cDNA collection, rather than one-by-one protocol, which is time- and labor-consuming. In the present report, we demonstrated its feasibility using a cDNA collection of the Arabidopsis DOF (DNA-binding with one finger) family (Yanagisawa 2002).

The 33 cDNAs for predicted CDSs of 36 DOF genes were amplified by PCR using the gene-specific primers and cloned into the Gateway entry vector, pENTR (Invitrogen, Carlsbad, CA, USA) as previously described (Tsujimoto-Inui et al. 2009). In this study, we selected 10 of the cDNA collection for transformation of T87 cells (Supplemental Table 1). The DOF CDS cDNA in the pENTR entry clones was recombined into a destination vector that is a Gateway-based binary vector, pK2GW7 (Karimi et al. 2002). This vector was provided from Plant Systems Biology (University of Ghent, Ghent, Belgium; http://www.psb.ugent.be/gateway/index.php). In this vector, DOF cDNAs were inserted downstream of cauliflower mosaic virus 35S (CaMV35S) promoter. Equally amounts of plasmids of the different entry clones for the 10 DOF cDNAs were mixed (50 ng $\mu 1^{-1}$ as final concentration). Using $100 \mathrm{ng}$ of pENTR plasmids and $150 \mathrm{ng}$ of $\mathrm{pK} 2 \mathrm{GW} 7$, the LR reaction was performed at half volume of the reaction solution that recommended in the manufacturer's instruction (Invitrogen, Carlsbad, CA, USA). E. coli TOP10 cells (Invitrogen, Carlsbad, CA, USA) were transformed using $3 \mu$ of the resultant reaction solution. After heat shock treatment, $25 \mu \mathrm{l}$ of cell suspension was transferred into $4 \mathrm{ml}$ of LB medium containing $100 \mu \mathrm{g} \mathrm{ml}^{-1}$ spectinomycin and incubated at $37^{\circ} \mathrm{C}$ overnight. Plasmids were isolated from $3 \mathrm{ml}$ of the cell suspension with a QIAprep Spin Miniprep Kit (Qiagen, Valencia, CA, USA). The inserted cDNAs into the plasmids were checked by $1.3 \%(\mathrm{w} / \mathrm{v})$ agarosegel electrophoresis and ethidium bromide $(\mathrm{EtBr})$ staining after PCR using $2.5 \mathrm{ng}$ of the plasmids, the gene-specific primer sets (Supplemental Table 1), and rTaq polymerase (Takara Bio Inc., Shiga, Japan).
Using the resultant plasmid mixtures, Agrobacterium tumefaciens LBA4404 was transformed according to the method previously described by Cindy and Jeff (1994). After $400 \mathrm{ng}$ of the plasmid mixture was added to $50 \mu \mathrm{l}$ of suspension of competent cells of A. tumefaciens LBA4404, the cells were transformed by freeze-thaw method. After heat shock treatment, $700 \mu \mathrm{l}$ of SOC was added and the cell suspension was incubated for $3 \mathrm{~h}$ at $28^{\circ} \mathrm{C}$ with shaking. One hundred microliters of the cell suspension was transferred into $4 \mathrm{ml}$ of YEB medium and cultured for 2 days at $28^{\circ} \mathrm{C}$ with shaking. Plasmids were isolated from $3 \mathrm{ml}$ of the culture with a QIAprep Spin Miniprep Kit (Qiagen, Valencia, CA, USA). The inserted cDNAs into the plasmids were checked again by $1.3 \%(\mathrm{w} / \mathrm{v})$ agarose-gel electrophoresis and $\mathrm{EtBr}$ staining after PCR using $2.5 \mathrm{ng}$ of the plasmids, the gene-specific primer sets (Supplemental Table 1), and rTaq polymerase (Takara Bio Inc., Shiga, Japan). A contamination of entry clone plasmids in the destination vector plasmids isolated from the pool of cells of $A$. tumefaciens was examined by PCR using a primer set, M13-M3 (GTAAAACGACGGCCAGT) and M13-RV (CAGGAAACAGCTATGAC), whose binding sites are existed in the entry vector but not in the destination vector. The remaining cell suspension was stored at $-80^{\circ} \mathrm{C}$ as a glycerol stock until used for transformation of Arabidopsis T87 cells.

Arabidopsis thaliana (L.) Heynh. ecotype Columbia suspension-cultured cells, line T87 (Axelos et al. 1992), were maintained according to the previously described methods (Tsujimoto-Inui et al. 2009). The Arabidopsis T87 cells were transformed by co-cultivation with the LBA4404 cells of $A$. tumefaciens harboring the pK2GW7 plasmids containing cDNAs for the 10 DOF genes according to the previously described methods (Tsujimoto-Inui et al. 2009). Cell clusters, which are resistant to kanamycin, were picked up and assigned to a single transgenic cell line. Total RNA was isolated from cells of every transgenic T87 cell line by the method described previously (Fukuda et al. 1991). To assess expression of the transgenes in each transgenic cell line, reverse transcriptase-mediated polymerase chain reaction (RT-PCR) was performed using $500 \mathrm{ng}$ total RNA and SuperScript III Reverse Transcriptase (Invitrogen Carlsbad, CA, USA), G-Taq (Hokkaido System Science Co., Ltd, Hokkaido, Japan) and a primer set specific for every DOF gene (Supplemental Table 1). Expression of all of the 10 DOF genes was examined in a line of callus. Transcripts of $\beta$-tubulin gene were also amplified as a constitutive expression control using a specific primer set (Supplemental Table 2). Each RT-PCR product was separated by $1.3 \%(\mathrm{w} / \mathrm{v})$ agarose-gel electrophoresis to visualize the amplified DNAs with EtBr staining.

The overview of proposed strategy for the batch processing protocol in this study is illustrated in Figure 
1. Entry clone plasmids selected from a collection of entry clones containing CDS cDNAs were pooled and simultaneously transferred to a destination vector for $A$. tumefaciens-mediated transformation of plant cells, i.e. a Gateway technology-based binary vector, by LR reaction. Suspension-cultured cells of Arabidopsis, line T87, were then transformed via A. tumefaciens cells, which were simultaneously transformed with a pool of the destination vector plasmids. Finally, kanamycinresistant calli were selected and individual callus were designated as transgenic cell lines. To validate the batched processing, the inserted DOF cDNAs into the destination vector were checked by PCR. Figure 2A shows the amplification of each of the 10 DOF cDNAs in a pool of the plant expression vector plasmids isolated from the E. coli transformants. Figure 2B, lanes 1-10, shows the presence of every DOF cDNA in a batch of

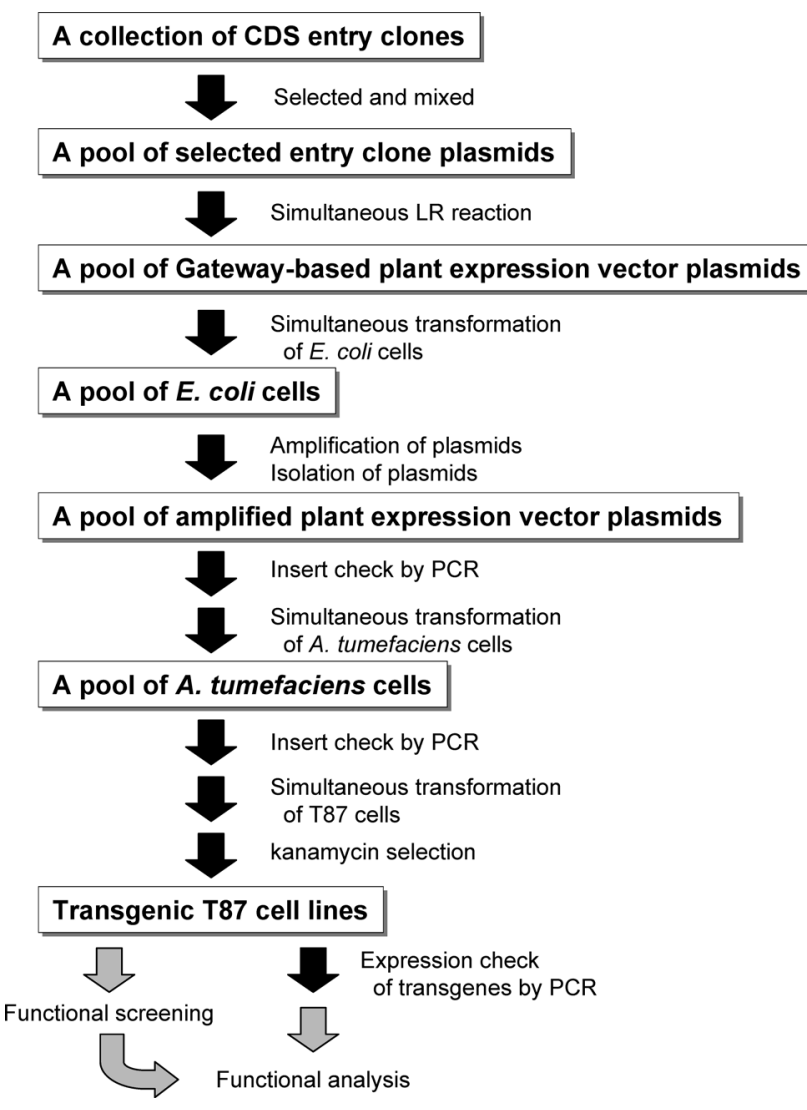

Figure 1. A summary of flow chart for a batch processing protocol for construction of binary vector plasmids using a collection of CDS cDNAs for a transcription factor family and subsequent A. tumefaciensmediated transformation of Arabidopsis T87 cells. Plasmids are selected from a collection of CDS entry clones. The pool of entry clone plasmids is simultaneously recombined into a Gateway-based plant expression vector, such as $\mathrm{pK} 2 \mathrm{GW} 7$, by a LR reaction. E. coli cells are transformed using the plasmid pool after LR reaction. A pool of the plant expression vector plasmids isolated from the $E$. coli transformants is then subjected to transformation of A. tumefaciens cells. Arabidopsis T87 cells are transformed by co-culture with the transgenic $A$. tumefaciens cells. The kanamycin-resistant cells are selected and used for further experiments. the plant expression vector plasmids isolated from $A$. tumefaciens cells. By contrast, no contamination of the entry clone plasmids was confirmed (Figure 2B, lane N). Thus, the batch processing from the LR reaction to the transformation of A. tumefaciens cells was properly performed. After transformation of Arabidopsis T87 cells using the batch of $A$. tumefaciens cells, we obtained 8 independent cell lines that are resistant to kanamycin. Then, we examined expression of each of the $10 \mathrm{DOF}$ genes in every cell line. As a control, expression of the DOF genes in the transgenic cell lines harboring CaMV35S:GUS, which were established by a conventional transformation method, was also examined. The results are shown in Figure 3. The specific overexpression of transgenes was observed in the several cell lines, i.e. line 1 and 6 overexpressing DOF3 gene, line 2 overexpressing DOF5, line 8 overexpressing DOF9 gene, and line 5 and 7 overexpressing DOF10 gene. No expression of genes for DOF1, 4 and 6 , and a constitutive expression of DOF7 and DOF8 genes were observed in both the DOF-transformed and the control cell lines. A constitutive expression of DOF2 was also observed in the control cell lines, but the reduced expression of DOF2 was observed in line 1, 5, 6, 7, and 8 , in which the overexpression of DOF3, DOF5, DOF9,

\section{A}
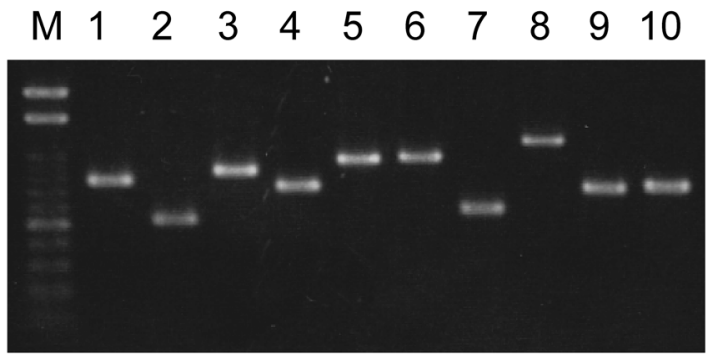

$\mathrm{B}$

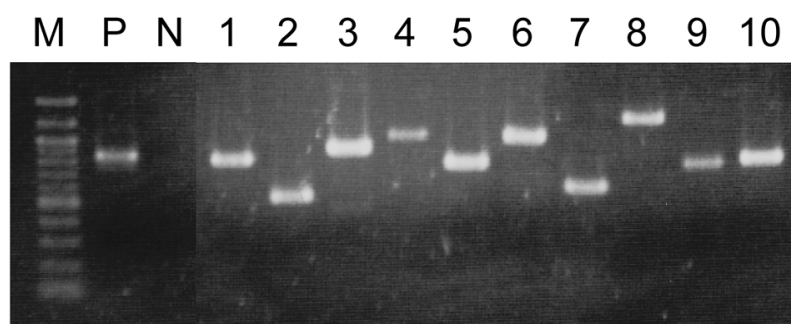

Figure 2. Confirmation of presence of CDS cDNAs during the batch processing. Insert DNA fragments in a pool of expression vector plasmids before (A) and after (B) the transformation of A. tumefaciens were confirmed by PCR using primer set specific for every DOF gene (Supplemental Table 1). Lane M, 100 bp DNA ladder; 1, DOF1; 2, DOF2; 3, DOF3; 4, DOF4; 5, DOF5; 6, DOF6; 7, DOF7; 8, DOF8; 9 , DOF9; 10, DOF10. P, a positive control for amplification of a cDNA fragment in an entry clone plasmid by PCR with M13-M3 and M13$\mathrm{RV}$; $\mathrm{N}$, no contamination of entry clone plasmids in the pool of $A$. tumefaciens cells was observed. 


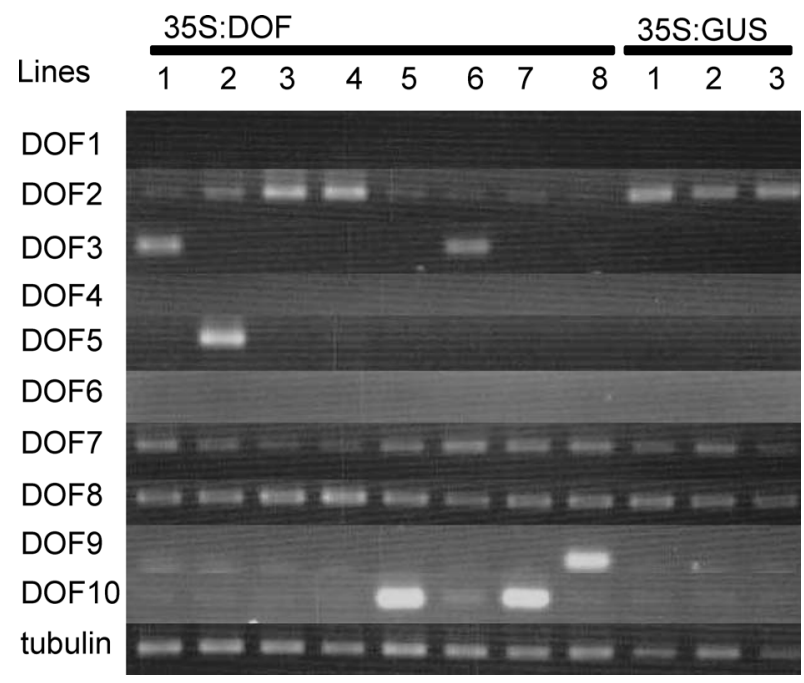

Figure 3. Analysis of expression of transgenes in established transgenic cell lines. Total RNA was prepared from the CaMV35S:DOF transgenic cell lines (lane 1 to 8) or CaMV35S:GUS transgenic cell lines as control cell lines (lane 1 to 3). PCR was carried out with 12.5-fold diluted aliquots of reverse transcriptase reactions. PCR primers specific to each DOF gene or a tubulin gene (Supplemental Table 2) were used. Basically, the PCR for the DOF genes was performed with 25 cycles and the results were presented here. Since no or quite low amplification of cDNA fragments for DOF1, DOF2, DOF4, DOF5, DOF6, DOF7, and DOF8 was detected, the PCR was performed again with 30 cycles. As a result, the specific bands for DOF2, DOF5, DOF7, and DOF8 but not for DOF1, DOF4, and DOF6 were confirmed. Therefore, the results of PCR with 30 cycles for DOF2, DOF5, DOF7, and DOF6 were presented. As an internal standard, expression of tubulin gene was also examined by PCR with 35 cycles.

or DOF10 was observed. No information is available to explain causes of such antagonistic expression of the DOF genes at this time. In the present study, the specific overexpression of a single DOF transgene was observed in at least 6 of 8 lines, and a transgenic callus obviously overexpressing multiple DOF transgenes was not obtained. These results show the potential utility of the present protocol to gain a clue for detailed functional analyses.

Here we demonstrated a simple and easy protocol for construction of plant expression vector plasmids using a collection of CDS cDNAs for a transcription factor family and subsequent $A$. tumefaciens-mediated transformation of Arabidopsis T87 cells using the pooled plasmids. Wieste et al. (2007) demonstrated similar attempts for comprehensive analysis of gene function of ERF transcription factor family in transgenic Arabidopsis plants and they showed that it is very useful for a screening approach to identify those members involved in particular developmental process or leading to enhanced stress responses. Transgenic cultured cells overexpressing transcription factors generated by our protocol may subsequently used for functional analyses, such as transcriptomic, proteomic and/or metabolomic analyses, and screening for drug and biotic/abiotic stress resistance. This protocol will be improved by using a large cDNA collection of transcription factor genes, which have been established by Gong et al. (2004), and other Gateway-compatible plant expression vectors for expression of a dominant-negative form, double-strand RNAs for RNAi, and GFP-tagged proteins, etc. Recently, Ogawa et al. (2008) reported a protocol for efficient and high-throughput vector construction to prepare Gatewaycompatible plant expression vector plasmids using RIKEN Arabidopsis full-length (RAFL) cDNA clones (Seki et al. 2002). Utilizing such cDNA collections in Gateway-compatible plant expression vector plasmids, the present protocol will be more useful to study functions of not only transcription factor genes but also all other genes in Arabidopsis. Furthermore, this protocol will be also applicable to other plant species.

\section{Acknowledgements}

The authors thank Dr. Shinozaki (RIKEN Plant Science Center, Yokohama, Japan) and RIKEN BioResource Center (Tsukuba, Japan) for providing Arabidopsis T87 cells, and Ms. S. Ito for her technical assistance. This study was supported by New Energy and Industrial Technology Development Organization (NEDO) as part of a project called 'Development of Fundamental Technologies for Controlling the Material Production Process of Plants'.

\section{References}

Arabidopsis Genome Initiative (2000) Analysis of the genome sequence of the flowering plant Arabidopsis thaliana. Nature 408: 796-815

Axelos M, Curie C, Mazzolini L, Bardet C, Lescure B (1992) A protocol for transient gene expression in Arabidopsis thaliana protoplast isolated from cell suspension cultures. Plant Physiol Biochem 30: 123-128

Bi Y-M, Zhang Y, Signorelli T, Zhao R, Zhu T et al. (2005) Genetic analysis of Arabidopsis GATA transcription factor gene family reveals a nitrate-inducible member important for chlorophyll synthesis and glucose sensitivity. Plant J 44: 680-692

Callard D, Axelos M, Mazzolini L (1996) Novel molecular markers for late phases of the growth cycle of Arabidopsis thaliana cellsuspension cultures are expressed during organ senescence. Plant Physiol 112: 705-715

Century K, Reuber TL, Ratcliffe OJ (2008) Regulating the regulators: the future prospects for transcription-factor-based agricultural biotechnology products. Plant Physiol 147: 20-29

Cindy RW, Jeff V (1994) Agrobacterium-mediated gene transfer to plant cells: cointegrated and binary vector systems. In: Gelvin SB, Schilperoort RA (eds) Plant Molecular Biology Manual Second Edition B1. Kluwer Academic Publishers, Dordrecht, pp 1-19

Davuluri RV, Sun H, Palaniswamy SK, Matthews N, Molina C et al. (2003) AGRIS: Arabidopsis gene regulatory information server, an information resource of Arabidopsis cis-regulatory elements and transcription factors. BMC Bioinformatics 4: 25

Fukuda Y, Ohme-Takagi M, Shinshi H (1991) Gene structure and expression of a tobacco endochitinase gene in susupensioncultured tobacco cells. Plant Mol Biol 16: 1-10 
Gong W, Shen YP, Ma LG, Pan Y, Du YL et al. (2004) Genomewide ORFeome cloning and analysis of Arabidopsis transcription factor genes. Plant Physiol 135: 773-782

Grotewold E (2008) Transcription factors for predictive plant metabolic engineering: are we there yet? Curr Opin Biotechnol 19: $138-144$

Guo AY, Chen X, Gao G, Zhang H, Zhu QH et al. (2008) PlantTFDB: a comprehensive plant transcription factor database. Nucl Acids Res 36: 966-969

Lee M-H, Kim B, Song S-K, Heo J-O, Yu N-I et al. (2008) Largescale analysis of the GRAS gene family in Arabidopsis thaliana. Plant Mol Biol 67: 659-670

Karimi M, Inze D, Depicker A (2002) GATEWAY vectors for Agrobacterium-mediated plant transformation. Trends Plant Sci 7: 193-195

Nakamichi N, Ito S, Oyama T, Yamashino T, Kondo T et al. (2004) Characterization of plant circadian rhythms by employing Arabidopsis cultured cells with bioluminescence reporters. Plant Cell Physiol 45: 57-67

Nakamura Y, Kimura A, Saga H, Oikawa A, Shinbo Y et al. (2007) Differential metabolomics unraveling light/dark regulation of metabolic activities in Arabidopsis cell culture. Planta 227: $57-66$

Ogawa Y, Dansako T, Yano K, Sakurai N, Suzuki H et al. (2008) Efficient and high-throughput vector construction and Agrobacterium-mediated transformation of Arabidopsis thaliana suspension-cultured cells for functional genomics. Plant Cell Physiol 49: 242-250

Overvoorde PJ, Okushima Y, Alonso JM, Chan A, Chang C et al. (2005) Functional genomic analysis of the AUXIN/INDOLE-3ACETIC ACID gene family members in Arabidopsis thaliana. Plant Cell 17: 3282-3300

$\mathrm{Qu}$ L-J, Zhu Y-X (2006) Transcription factor families in Arabidopsis: major progress and outstanding issues for future research. Curr Opin Plant Sci 9: 544-549

Riaño-Pachón DM, Ruzicic S, Dreyer I, Mueller-Roeber B (2007)
PlnTFDB: An integrative plant transcription factor database. BMC Bioinformatics 8: 42

Riechmann JL, Heard J, Martin G, Reuber L, Jiang C et al. (2000) Arabidopsis transcription factors: genome-wide comparative analysis among eukaryotes. Science 290: 2105-2110

Seki M, Narusaka M, Kamiya A, Ishida J, Satou M et al. (2002) Functional annotation of a full-length Arabidopsis cDNA collection. Science 296: 141-145

Stolc V, Samanta MP, Tongprasit W, Sethi H, Liang S et al. (2005) Identification of transcribed sequences in Arabidopsis thaliana by using high-resolution genome tiling arrays. Proc Natl Acad Sci USA 102: 4453-4458

Takahashi S, Katagiri T, Hirayama T, Yamaguchi-Shinozaki K, Shinozaki K (2001) Hyperosmotic Stress Induces a Rapid and Transient Increase in Inositol 1,4,5-Trisphosphate Independent of Abscisic Acid in Arabidopsis Cell Culture. Plant Cell Physiol 42: 214-222

Tsujimoto-Inui Y, Naito Y, Sakurai N, H Suzuki, Sasaki R et al. (2009) Functional genomics of the Dof transcription factor family genes in suspension-cultured cells of Arabidopsis thaliana. Plant Biotechnol 26: 15-28

Uno Y, Furihara T, Abe H, Yoshida R, Shinozaki K et al. (2000) Arabidopsis basic leucine-zipper transcription factors involved in an abscisic acid-dependent signal transduction pathway under drought and high-salinity conditions. Proc Natl Acad Sci USA 97: 11632-11637

Weiste C, Iven T, Fischer U, Onate-Sanchez L, Droge-Lase W (2007) In planta ORFeome analysis by large-scale overexpression of GATEWAY-compatible cDNA clones: screening of ERF transcription factors involved in abiotic stress defense. Plant J 52: 382-390

Yanagisawa S (2002) The Dof family of plant transcription factors. Trend Plant Sci 7: 555-560

Zhang JZ (2003) Overexpression analysis of plant transcription factors. Curr Opin Plant Biol 6: 430-440 